\title{
A menu for safe (food) keeping
}

\section{MARGARET REICH \\ Senior Staff Editor}

"Bad cooks - and the utter lack of reason in the kitchen - have delayed human development longest and impaired it most." - Nietzsche, German philosopher, 1886.

Although that quote appeared more than 100 years ago in Beyond Cood and Evil, it serves as an indirect reminder that improper food handling and preparation are the perfect ingredients for possible food-borne illness. The recent Chilean grape poisoning incident and Alar apple scare have prompted consumers to tread cautiously through the produce aisle. Yet experts from the Food and Drug Administration (FDA) and the Institute of Food Technologists, among others, cite naturally occurring microbial contamination as a greater threat to food safety than pesticides.

Specifically, the Institute of Food Technologists, whose members consist of microbiologists, toxicologists, veterinarians, and food scientists, issued a report to Congress recently which said, "Current scientific evidence indicates that any health risk associated with pesticide residues is negligible and is of a magnitude less than that of food-borne diseases such as salmonella and shigella."

At least from a financial standpoint, this point seems well documented: Diarrhea due to food-borne illness costs the United States $\$ 1$ to $\$ 10$ billion annually in lost productivity and wages, medical costs, product embargos, and food destruction. Approximately one in ten Americans will suffer from diarrhea related to food-borne illness this year.

Most types of food poisoning are not lifethreatening to healthy adults. However, a bout with tainted fare proves more hazardous for infants under 3 months old, the elderly, pregnant women, and people whose immune systems have been weakened because of disease (cancer and AIDS patients, for example) or medication. Individuals taking antibiotics or antacids have lower resistance, which makes them more susceptible to food poisoning, as well.
With that in mind, an ounce of prevention is worth its weight (by volume) in Peptobismol. The nose knows when good food has gone bad. Yeast and mold growths serve as visible warning signs of inedible victuals. Above all, when in doubt, throw it out!

On the other hand, the bacteria that account for food poisoning (not spoilage) are a shy group; they leave no visible clues. Even a careful cook cannot tell if tonight's chicken features a side order of Salmonella bacteria. Fortunately, most bacteria and other microorganisms can be killed with proper handling, cooking, or freezing. But before we discuss these defensive techniques, let's examine a few culinary culprits more closely.

\section{Staphylococcus aureus}

Called staph for short, Staphylococcus aureus can take credit for causing most cases of food poisoning in the United States. Ironically, each of us carries this bacteria in our noses and on our skin. Skin infections provide fertile ground for staph.

Without proper hygiene, staph can easily be transferred to food. Hands and utensils should be scrupulously clean before meal preparation. Custard or cream-filled pastries, milk, cheese, processed meat, fish, and starchy salads (potato, macaroni, pasta) are especially vulnerable to staph contamination. Cooking does not destroy all the bacteria. As long as food is consumed promptly after cooking, food poisoning is unlikely. However, if edibles are left out in warm temperatures $\left(100^{\circ} \mathrm{F}\right)$, the residual bacteria, which survived the initial cooking, will "revive" and produce a toxin.

Three to six hours after eating a staph-infected dish, the unknowing consumer will suffer nausea, vomiting, and diarrhea. These symptoms may be complicated by abdominal cramps, headache, fever, and possibly bloody stool. Fortunately, most healthy people recover within a day or two. 


\section{Salmonella}

Unpasteurized milk, raw eggs (particularly Grade A), and undercooked or raw poultry and meats frequently play host to this microorganism. Approximately one third of all raw poultry sold in this country may be contaminated with Salmonella bacteria, according to the Department of Agriculture.

Feed contaminated with Salmonella finds its way into the animal's digestive tract where the bacteria set up housekeeping in the intestines. Salmonella may be transmitted through the animal's feces. For example, in the mechanical defeathering process, infected feces may be spewed onto the feathers. When the "rubber fingers" remove the feathers, they may inadvertently spread the bacteria onto the skin. Likewise, Salmonella may be dispersed when the chicken's internal organs are removed.

Researchers at the United States Department of Agriculture (USDA) are studying methods to prevent the Salmonella bacteria from colonizing in the intestines of chickens. A natural sugar, D-mannose, seems to prevent some bacteria from residing in the intestines of rats and mice. When tested on broiler chickens, the results proved promising. Further research and FDA approval are necessary before this method can be used in poultry production.

Egg shells produced by a contaminated chicken may also be tainted. Furthermore, if Salmonella bacteria are present in a hen's ovary, the egg yolk can be contaminated before shell formation. So, even though the shell undergoes washing with disinfectants during processing, it remains contaminated in this instance.

Salmonella microorganisms account for the vomiting and diarrhea likely to occur 12 to 48 hours after invading the body. Fever makes a less-thanpleasant situation more miserable. Expect symptoms to persist for two to seven days. Rest, fluids, and a bland diet is the best prescription in most cases.

\section{Clostridium perfringens}

Cafeteria food has a reputation for being somewhat inedible. Thanks to Clostridium perfringens, or simply perfringens, that reputation proves true. This microorganism exists throughout the environment, including animal and human intestines. Perfringens features normal cells and the hardy spore cells. Under "perfect" conditions-incomplete cooking and rewarming; large quantities of food left at room temperatures for more than two hours-spore cells become normal cells. These multip[ly quickly and produce a toxin responsible for food poisoning. Cooked beef, turkey, dressing, stews, and casseroles are prime candidates for such contamination.

Diarrhea, occasionally accompanied by vomiting, probably will occur sometime within 8 to 16 hours after tainted food has been consumed. The suffering should subside within one to four days.

\section{Botulism}

A rare form of food poisoning, botulism can be deadly. Clostridium botulinum are spores found in soil and water; they flourish with little need for oxygen. The canning process gets rid of air from food. But if the canned food has not been cooked at high enough temperatures to kill these spores, the spores revert to normal botulinum cells. These cells produce a toxin, which assaults the nervous system.

Improperly canned vegetables (both commercial and homegrown), smoked meats, vacuum-packed fish, and other improperly canned goods prove dangerous. Vegetables with low acidity - green beans, corn, beets, peas-have a better chance of picking up botulinum spores from the soil. In infants, botulism has been linked with honey.

Recently, the FDA recommended consumers avoid garlic in oil that does not contain citric acid or phosphoric acid. Whether or not the homemade or commercially produced product contains these antimicrobial agents, it should be refrigerated.

Symptoms - double vision, dry throat and mouth, and difficulty swallowing and speaking - appear approximately 12 to 36 hours after ingesting the contaminated food. In the most severe instance, the toxin affects the nerves that stimulate breathing, and suffocation ensues.

Without treatment, the mortality rate is high$30 \%$ to $70 \%$. An antitoxin that has reduced the number of botulism-related deaths can be administered. However, nerve damage may persist.

Fortunately botulism gives cooks fair warning. Milky or thick liquids that should be clear; cracked 
jars, loose lids, swollen or dented cans automatically signal trouble. Home-canned vegetables deserve extra attention. Never taste any suspicious looking food. Carefully wrap the food in its original container in plastic and brown bags. Place the tightly wrapped package in a garbage can, covered securely with a lid. (See page $\mathbf{6 9 8}$ for further information on contacting health authorities.)

\section{Listeria}

Found in the soil, vegetables, fruits, fish, meat, poultry, and dairy products, Listeria moncytogenes bacteria are particulary hardy. Traditional methods of killing bacteria and other microorganismsrefrigeration, heat, salt, nitrite, and acid- pack no punch against this formidable microbe.

One of the largest US outbreaks of Listeria food poisoning occurred in California in 1985. At that time, soft-style Mexican cheese was implicated. But, raw cabbage, unpasteurized cow's milk, and other dairy products can harbor this bacteria, too.

Listeria monocytogenes causes an infection called listeriosis. Pregnant women and others whose immune system is weakened are at special risk. Symptoms in pregnant women resemble the flu. Likewise, meningitis - inflammation of membranes encasing the brain and spinal cord-is a common manifestation of listeriosis in adults. This infection warrants professional medical care.

Unborn children of infected mothers also are in danger. If the microorganism crosses the placenta, the fetus can be infected. This may cause premature labor and possibly fetal death (miscarriage).

The best way to prevent this infection is to cook all meats, fish, and eggs thoroughly and to avoid drinking unpasteurized milk.

\section{Trichinosis}

Although a parasite and not a bacterium, Trichinella spiralis can cause gastronomical complications similar to those previously mentioned. But thanks to consumer awareness of this roundworm, fewer than 100 cases of trichinosis are reported to the Centers for Disease Control (CDC) each year. Most cooks realize the need for thoroughly cooking pork to kill this parasite. Also, animals once fed garbage, are now eating special feed in many instances. (If garbage is still part of the animals' diet, it has been rendered. But, feeding procedures are not uniform industrywide, so careful cooking remains important to prevent contamination.)

Symptoms don't always manifest themselves. If they do, diarrhea, nausea, and vomiting occur about a day or two after contaminated meat is eaten. Such discomforts come courtesy of the worm burrowing itself into the intestinal wall and depositing larvae. The larvae make their way into the bloodstream and on to other tissues and organs. But only those that reach the skeletal muscle tissues survive. The roundworms find the diaphragm, tongue, and the pectoral and eye muscles particularly cozy.

Once the parasites set up residence about a week or two later, the victim might experience swollen eyelids due to water retention (edema). Muscle aches and difficulty swallowing or talking may follow.

Most people recover on their own without major complications. Aspirin and other anti-inflammatory agents may be prescribed for pain and swelling.

\section{When to get help}

If these or other food-borne symptoms persist despite home remedies - rest, fluids, bland diet-don't hesitate to seek professional help. Definitely get help if you have trouble swallowing, speaking, or breathing. A temperature higher than $100^{\circ} \mathrm{F}$ that persists for more than three days can be serious, too. It's wise to check with your doctor if you experience more than eight bowel movements in a day, have not been producing normal amounts of urine, or if you are extremely thirsty or have dry mouth.

Now that some of these better-known microorganisms have been uncovered, it's time to learn how to foil food poisoning.

\section{The chef's creed}

Keeping food contamination under wraps depends on keeping edibles hot or cold and making sure the kitchen remains spotless. NEVER defrost food at room temperature. Thawing in the refrigerator is the safest method. Above all, avoid the internal temperature danger zone $-60^{\circ} \mathrm{F}$ to $125^{\circ} \mathrm{F}$. Bacteria grow rapidly in this climate and can produce toxins. The higher the cooking temperature, the less time is required for killing bacteria. Food that has been prepared to 
reach high internal temperatures $\left(165^{\circ}\right.$ to $\left.212^{\circ} \mathrm{F}\right)$ should be served promptly.

Buffet-style food poses special problems. While chafing dishes and steam tables are designed to keep food at internal holding temperatures between $140^{\circ}$ and $165^{\circ} \mathrm{F}$, often these devices fail to distribute heat evenly. Food divided into smaller portions permits thorough heating. And regardless of what's on the menu, never leave hot food out for more than two hours. This only serves as an invitation for microbial dinner "guests."

Thorough, uninterrupted cooking is paramount. The chart on this page lists temperatures recommended by the USDA for safe cooking. Frozen foods will need to be cooked about one and a half times longer than fresh meat and poultry.

Leftovers should be refrigerated or frozen promptly. Don't let them cool on the counter beforehand. Lots of leftovers? Remember to divide them into smaller portions before storing. When it's time for reheating, cover leftovers to help retain moisture and provide even heat throughout the dish. Gravies brought to a rolling boil are a safe bet to eat.

\section{Microwave menus}

Cooking in the microwave requires special precautions because "cold spots" can exist inside this appliance. That's why food pieces should be evenly arranged under a covered dish. (Plastic wrap or a glass cover will do.) The food, as well as the entire dish, should be turned periodically for thorough cooking.

A temperature probe inserted in several different areas of the meat or poultry (not near bone or fat) should read $160^{\circ} \mathrm{F}$ for red meat and $180^{\circ}$ for poultry. Don't ignore the standing time noted in recipes.
Those minutes in which a cooked dish stands covered with foil outside the microwave create a steamy atmosphere for the food to finish cooking.

Not every food cooks well in the microwave. Stuffed fowl (chicken and turkey) can turn foul. Explains Susan Templin, home economist and supervisor of the USDA'S Meat and Poultry Hotline: "It just takes too long for the stuffing to reach a safe enough temperature in the microwave. We recommend cooking the stuffing separately." If stuffing the bird overnight sounds like a timesaving idea for convent ional cooking, think again. Notes Templin, "The poultry juices may carry bacteria like Salmonella. The stuffing itself is such a prime medium for bacterial growth; it's moist and a carbohydrate [starchy]. The stuffing just might not get cold enough fast enough in the refrigerator, and then hot enough fast enough in the oven to kill the bacteria."

\section{Egg-ceptional preparation}

Those who enjoy eggs over easy or sunny-side up take note: Eating a runny egg could be chancey, especially for persons in high risk groups. But Templin advises, "If a normal, healthy, middle-aged person wants to consume a Caesar salad or sunny-side up egg, and if he or she uses fresh, refrigerated, shellsintact, clean eggs, prepared in a sanitary manner, and consumes it right away, the risk is very, very small."

In preparing scrambled eggs, it's best to use the mixture promptly to prevent bacterial growth. If a large brood sits down at the breakfast table, prepare smaller amounts separately rather than mixing a large bowlful at once.

Cooking hard-boiled eggs requires covering the 
eggs with water and bringing it to a boil. After about 15 or 20 minutes of boiling, remove eggs from heat and pour out the hot water. The eggs should be rinsed-not soaked-in cold water. Soaking in cold water makes the shell more permeable, increasing the chance of bacterial invasion. Placed in a shallow bowl, eggs can be air dried in the refrigerator. Hardboiled eggs (shelled or peeled) keep for about one week in the refrigerator.

\section{The fish story}

Many cooks are like a fish out of water when it comes to preparing seafood properly. With that in mind, Beverly Barton, a home economist with the National Marine Fisheries Service, suggests cooking fish to an internal temperature of $140^{\circ}$ to $160^{\circ} \mathrm{F}$ until the fish is flaky. She explains, "The fish goes from being translucent in its raw state to opaque [when cooked]. When the thickest part of the fish reaches the opaque state, it will flake easily." (Raw fish-including sushi is best avoided, particularly by high-risk individuals.)

Preparing steamed shellfish, clams, and oysters can be a little trickier. Barton says such seafood is done after about three or four minutes when the shells open and that steaming much longer makes the seafood tough and inedible. However, the FDA says most viruses are killed after four to six minutes. Yet, both agree a fresh product from the outset determines safety. (This rule applies to all food, but especially for easily perishable seafood.) So no matter how attractive those fish prices on the back of a roadside truck appear, head for the nearest reputable fish or supermarket, instead.

Once at the supermarket, keep these fish freshness tips in mind:

- For whole fish, make sure the head is still on.

- Bulging eyes are a good sign, while sunken, graying eyes indicate aging.

- Scales, if present, should adhere tightly to skin. A fresh fish has shiny skin. Flesh that springs back after being touched serves as a good clue to freshness. (Actually touching fish yourself probably isn't allowed at the supermarket, but this test can be done at home after unwrapping.)

- Pink gills are a sign of a healthy, fresh fish. But deep red or gray gills mean trouble.

- Fillets should look moist. If the muscles seem to separate into flakes, look for another fish.

- Choose tightly closed shellfish. A gaping shell means the fish is dead. That's about as stale as you can get!

- A strong fishy smell indicates that species is past its prime. Fresh fish has a natural sea odor.

\section{Bringing home the bacon, eggs, etc...}

After a trip to the grocery store, don't hesitate to refrigerate. Because cold provides protection against most bacterial growth, make sure your refrigerator maintains a $40^{\circ} \mathrm{F}$ temperature or lower. Likewise, the freezer should register $0^{\circ} \mathrm{F}$ or lower. Make sure neither section is overpacked. Otherwise there won't be adequate air circulation.

\section{Refrigerating}

Rewrap only torn packages in plastic, foil, or waxpaper. Extra handling simply affords bacteria additional opportunities to invade food. Poultry, hamburger, or fish intended for dinner in a day or two can be stored safely in the refrigerator. Pork lasts three to five days. Keep these in the coldest partthe lower shelf toward the back of the meat tray.

Refrigerate eggs in their original carton. Whole eggs can be stored for up to five weeks, but they taste best if used within a week.

Contrary to popular belief, commerical mayonnaise does not contribute to food poisoning. Because lemon juice or other acid flavor, as well as salt, is added to mayonnaise, bacterial growth is thwarted. After opening, it can be safely kept on the refrigerator door shelf for a maximum of two months. However, salads containing mayonnaise should not be frozen. The mayonnaise separates from the other salad ingredients and on thawing creates an oily mess.

\section{Freezing}

Store frozen meat, poultry, or fish in freezer bags or foil to prevent freezer "burn." Although this white covering doesn't harm food, it can make meats less tender on cooking. Date these packages so you know how long to keep them.

Generally, fresh meats freeze safely for six to 12 months; hamburger lasts three to four months. Bacon 
lasts one month in the freezer, while ham can be stored for up to two months.

Whole chicken and turkey can be frozen for up to a year, while chicken pieces last nine months. Turkey pieces should be gobbled before six months lapse in the freezer.

Fresh fish can be frozen, too. But fattier fish (salmon, tuna) turns rancid quicker because the fat breaks down and oxidizes. To be safe, freeze fresh fish no longer than four months, but even that's testing the time limit. As might be expected, lean fresh fish tightly wrapped-cod, flounder-lasts longer (six to eight months).

Yet, proper storage is futile without good hygiene.

\section{Cleaning the cook's cupboard}

Before stirring, blending, tossing, or slicing, remember to wash your hands. This is especially important after using the bathroom. Washing hands after cutting vegetables but before dicing meat helps prevent cross-contamination. Wear gloves if you have a cut or other skin infection on your hands.

Hands aren't the only vehicle for contaminating food. That innocent looking wooden cutting board actually serves as a "hang out" for bacteria.

Microorganisms hide in the knife-carved crevices. So, if you cut up chicken, for example, and then decide to dice carrots on the same cutting board, Salmonella bacteria could "jump ship" to the carrots. Opt for an acrylic cutting board that can be placed in the dishwasher for thorough cleaning after each use.

Using the same knife for both foods - without washing it-carries bacteria from one source to another as well. Any utensils, dishes, appliances, or countertops that come in contact with raw food should be cleaned with hot soapy water to prevent cross-contamination. Serving cooked meat on the same unwashed dish in which the raw meat sat prior to preparation spells trouble.

Another precautionary measure is to rinse chicken in cold water before any cutting begins. Likewise, scrubbing vegetables with a bursh and rinsing under running water before dicing not only helps remove dirt and bacteria, but surface pesticides, too. (Don't forget to rinse the sink with hot water later to remove bacteria.)
Bacteria congregate in washclothes, sponges, and towels. Frequent laundering makes sense. If your kitchen sponge has been around longer than your mother-in-law, buy a new one!

With warm weather here, these hygiene practices are particularly important along with preparation and storage. (See below for picnic pointers.) Although taking steps to prevent food contamination won't turn a bad cook into a gourmet, at least the chef will be healthy.

\section{Safe, portable food}

Whether you're picnicking on the weekends or just brown-bagging lunch during the week, food to go requires special care, particularly during warm weather. While the same preparation and hygiene principles apply to portable as well as home food, properly storing picnic fare is even more important. Use a well-insulated cooler packed with ice or a freeze-pack insert. Cold beverage cans offer additional "refrigeration."

Keep the cooler in the shade, if possible. Return leftovers to the cooler as soon as everyone is done eating. There food can be stored safely up to four or five hours. On the way home, keep the cooler in the car rather than in the heat-retaining trunk.

Packing everyday lunches poses similar problems. While most 9-to-5 workers don't carry a cooler to the office, lunches can be kept cold through other means. Keep sandwiches in the refrigerator until just before leaving home. Add chips and cookies, which tend to go limp in the refrigerator, in the morning. Fresh fruit and vegetables make the trip to the office quite well. At work, refrigerate food.

If no refrigeration is available, freeze sandwiches at home the night before. Course breads work best here because they don't turn mushy upon thawing. Add lettuce, tomato, and mayonnaise at lunch. (These condiments don't freeze well.)

A thermos offers the best protection for milk and juice. Boxed juices that require no refrigeration might be another option. Wash lunchboxes and laminated bags daily. Brown baggers, use a clean bag each day.

These portable food tips should make eating outside the home more enjoyable and safe. Bon appetit ! 
(All material in the Patient Health Guide is reviewed by JAOA physician referees.)

\section{References:}

The ABCs of food storage. By Roger W. Miller. FDA Consumer, vol 20 pp 12-14, March 1986.

Covering the concerns for fresh produce. By Karen S. Peterson and Michelle Healy. USA Today (Life Section), pp 1-2, March 17, 1989.

Current Medical Diagnosis \& Treatment 1988. Edited by Steven A. Schroeder, Marcus A. Krupp, and Lawrence M. Tierney, Jr. Appleton \& Lange, Norwalk, Conn, 1988.

Do you dare to eat a peach? By Margaret Carlson. Time, pp 24-30, 35-36, March 27, 1989.

Handling eggs safely at home (consumer bulletin). United States Department of Agriculture, Food and Drug Administration, Sept 1988.

How safe is safe? by Ken Flieger. FDA Consumer, vol 22, pp 16-17, Sept. 1988.

How to spot unsafe food. Family Circle, pp 66, April 25, 1989.

The International Thesaurus of Quotations. Compiled By Rhoda Thomas Tripp. Harper \& Row Publishers, New York, 1987.

Invisible villains: Tiny microbes are biggest food hazard. By Marian Segal. FDA Consumer, vol 22, pp 9-11, July-Aug 1988.

Kitchen alert: The right way to store and prepare food. By Dr. Brian L.G. Morgan. Ladies' Home Journal, vol 104, p 50, 52, 54.

Listeria: Battling back against one 'tough bug.' By Karen Skinner, PhD. FDA Consumer, vol 22, pp 12-17, July-Aug 1988.

Maybe it was something you ate. By Penny Ward Moser. Saturday Evening Post, vol 259, pp 46-49, May-June 1987.

The Merck Manual of Diagnosis and Therapy. Edited By Robert Berkow, et al. Ed 15. Merck Sharp \& Dohme Research Laboratories, Rahway, NJ 1987

Mother Nature's regulations on food safety. By Roger W. Miller. FDA Consumer, vol 22, pp 52-54, November 1988.

Natural sugar cuts Salmonella in broilers. By Sandy Miller Hays. Agricultural Research, vol 37, pp 16-17, March 1989

Officials call microbes most urgent food threat. By William K. Stevens. The New York Times (Science Times), pp 17, 19, March 28, 1989.

Rx for food safety. By Mary Ann Parmley. Food News for Consumers, vol 6, pp 10-11, Spring 1989.

Red flags on food safety. By Linda Russell. Food News for Consumers, vol 5, pp 10-11, Winter 1989.

The Safe Food Book: Your Kitchen Guide (booklet). By Mary Ann Parmely, Stanley S. Green, and Carl S. Custer. United States Department of Agriculture, Food Safety and Inspection Service, Washington, DC, 1985.
Safe food to go- for lunches \& picnics. United States Department of Agriculture, pp 1-6, May 1988

Update: Salmonella enteritidis infections and grade A shell eggs-United States. Morbidity and Mortality Weekly Report, vol 37, p 490, 495-496, August 19, 1988

Weighing the risks of the raw bar. By Carol Ballentine. FDA Consumer, vol 20, pp 21-23, Sept 1988

When it comes to stylish sushi, it's safer to be square. By Evelyn Zamula FDA Consumer, vol 21, pp 19-21, Feb 1987.

\section{Steamed over tonight's dinner?}

Cooks with questions can call the USDA Meat and Poultry hotline weekdays from 10:00 am to 4:00 pm (Eastern Time) at 1-800-535-4555. (In Washington, DC, phone 447-3333.) Both numbers are accessible by Telecommunications Devices for the Deaf. Home economists answer questions concerning food preparation, handling, and storage. They can help consumers better understand meat and poultry labels.

Does that beef or poultry look suspicious? Before calling the hotline, refrigerate food in the original container, if possible, and contact the store where it was purchased. If you ate the suspected food at a large gathering or restaurant, or purchased it from a sidewalk vendor, contact local health officials. They will need to know the name and address of the store or restaurant in which the suspected food was purchased or eaten. Any lot numbers or government inspection stamps on the packaging will help in finding the original source of food poisoning. Experts at the USDA meat and poultry hotline can refer you to the proper health agencies for reporting these incidences, too.

The hotline can be reached by mail. Write to:

The Meat and Poultry Hotline

USDA-FSIS, Rm. 1165-S

Washington, DC 20250 


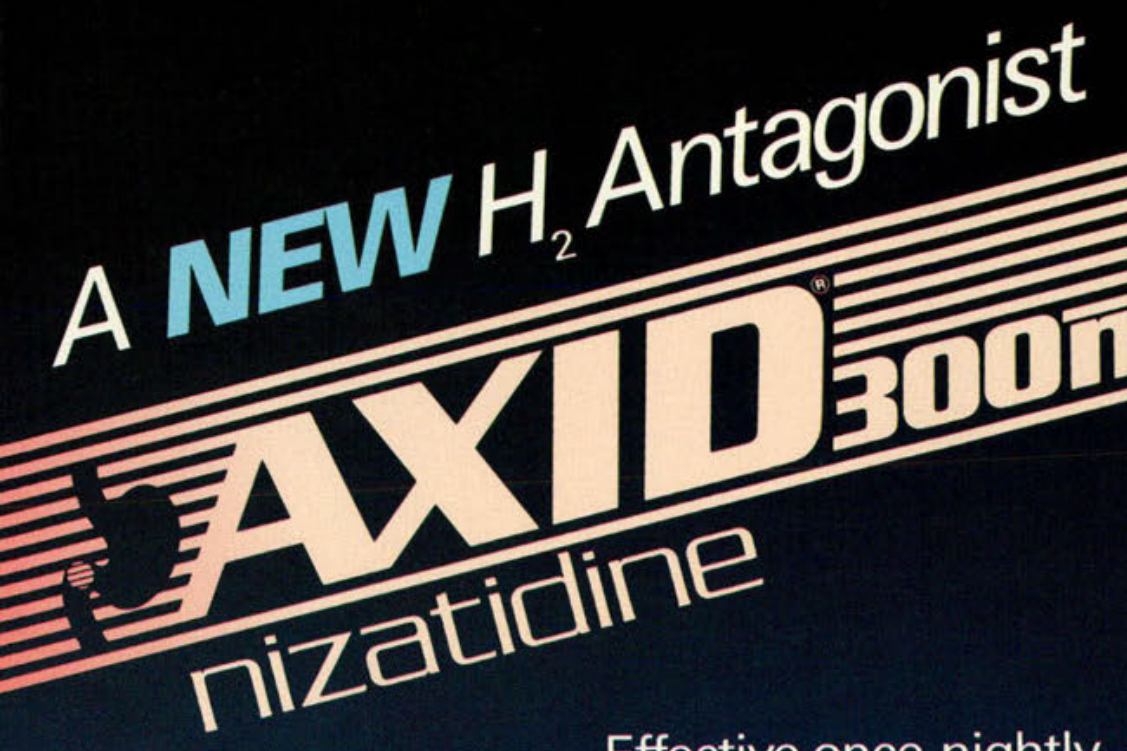

Effective once-nightly duodenal ulcer therapy available in a Unique Convenience Pak for better patient compliance

\section{AXID}

Briet Summary. Consult the package insert for prescribing intormation. Indications and Usage: Axid is indicated for up to eight weeks for the treatment of active duodenal ulcer. In most patients, the ulcer will heal within four weeks. Axid is indicated for maintenance therapy for duodenal ulcer patients, at The consequences of continuous therapy with Axid for longer than one year The consequences
are nown.

Contraindication: Axid is contraindicated in patients with known hypersensitivity to the drug and should be used with caution in patients with hypersensitivity to other $\mathrm{H}_{2}-\mathrm{receptor}$ antagonists.

Precautions: General-1. Symptomatic response to nizatidine therapy does not preclude the presence of gastric malignancy

2. Because nizatidine is excreted primarily by the kidney, dosage should be reduced in patients with moderate to severe renal insufficiency. been done Part of the dose of nizatidine is metabolized in the liver. In patients with nermal renal function and uncomplicated hepatic dystunction, the disposition of nizatidine is similar to that in normal subjects.

Laboratory Tests - False-positive tests for urobilinogen with Multistix* may occur during therapy with nizatidine.

Drug Interactions - No interactions have been observed between Axid and theophylline, chlordiazepoxide, lorazepam, lidocaine, phenytoin, and wartarin. Axid does not inhibit the cytochrome P-450-linked drug-metabolizing enzyme system; therefore, drug interactions mediated by inhibition of hepatic $\mathrm{mg}$ ) of aspirin daily, increases in serum salicylate levels were seen when nizatidine, $150 \mathrm{mg}$ bi.d. was administered concurrently. Carcinogenesis, Mutagenesis, Impairment of Fertility - A two-year ora carcinogenicity study in rats with doses as high as $500 \mathrm{mig} / \mathrm{kg} / \mathrm{cry}$ (about 80 carcinogenic effect. There was a dose related increase in the density of enterochromatfin-like ( $\mathrm{ECL}$ ) cells in the gastric oxyntic mucosa. In a two-year study in mice, there was no evidence of a carcinogenic effect in male mice: although hyperplastic nodules of the liver were increased in the high dose males compared to placebo. Female mice given the high dose of increases in hepatic carcinoma and hepatic nodular hyperplasia with n numerical increase seen in any of the other dose groups. The rate of hepatic carcinoma in the high dose animals was within the historical controf timits seen for the strain of mice used. The flemale mice were given a dose larger than the compared to concurrent controls, and evidence of mild liver iniury (transaminase elevations). The occurrence of a marginal finding at high dose only in animals given an excessive, and somewhat hepatotoxic dose. with no evidence of a carcinogenic eflect in rats, male mice, and a negative mutagenicity battery is not considered evidence of a carcinogenic potential for Axid. Axid was not mutagenic in a battery of tests performed to evaluate its potential genetic toxicity, including bacterial mutation tests, unscheduled DNA synthesis.
sister chromatid exchange, and the mouse lymphoma assay. In a two-generation, perinatal and postnatal, fertility study in rats, doses of
nizatidine up to $650 \mathrm{~m}$. $k$ kg/day produced no adverse effects on the reproductive pertormance of parental animals or their progeny. studies in rats at doses up to 300 times the human dose, and in Dutch Belted abbits at doses up to 55 times the human dose, revealed no evidence of impaired lertility or teratogenic effect; but, at a dose equivalent no dose, treated rabbits had abortions, decreased number of live fetuses, and depressed fetal weights. On intravenous administration to pregnant New Zealand White rabbits, nizatidine at $20 \mathrm{mg} / \mathrm{kg}$ produced cardiac enlargement, coarctation
of the aortic arch, and cutaneous edema in one fetus and at $50 \mathrm{mg} / \mathrm{kg}$ it produced ventricular anomaly, distended abdomen, spina bifida, hydrocephaly, and entarged heart in one fetus. There are, however, no adequate and well-controlled studies in pregnant women. It is also not known whether nizatidine can cause fetal harm when administered to a pregnant woman or can affect reproduction
capacity. Nizatidine should be used during pregnancy only if the potential benefit

justifies the potential risk to the fetus.
Nursing Mothers - Nizatidine is secreted and concentrated in the milk of lactating rats. Pups reared by treated lactating rats had depressed growth rates. Although no studies have been conducted in lactating women, nizatidine is assumed to be secreted in human milk, and caution should be exercised when
nizatidine is administered to nursing mothers.

Pediatric Use-Safety and effectiveness in children have not been established. Use in Elderly Patients - Ulcer healing rates in eiderly patients are similar to those in younger age groups. The incidence rates of adverse events and Age alone may not be an important factor in the disposition of nizatidine. Eiderly patients may have reduced renal function.

Adverse Reactions: Clinical trials of nizatidine included almost 5,000 patients given nizatidine in studies of varying durations. Domestic placebo-controlled Among the more common adverse events in the domestic placebo-controlled trials, sweating ( $1 \%$ vs $0.2 \%)$, urticaria $(0.5 \%$ vs $<0.01 \%)$, and somnolence $(2.4 \%$ vs $1.3 \%)$ were significantly more common in the nizatidine group. A
variety of less common events was also reported: it was not possible to

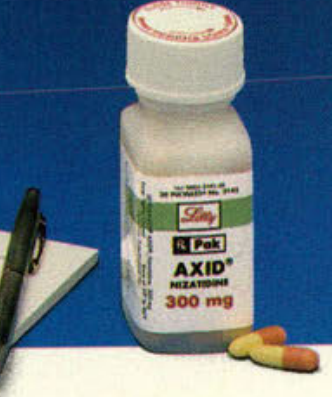

determine whether these were caused by nizatidine.

Hepatic - Hepatocellular iniury. evidenced by elevated liver enzyme tests (SGOT [AST], SGPT [ALT], or alkaline phosphatase), occurred in some patients possibly or probably related to nizatidine. In some cases, there was marked elevation of SGOT. SGPT enzymes (greater than $500 \mathrm{TU} / \mathrm{L}$ ), and in a single instance. SGPT was greater than 2,000 IU/L. The overail rate of occurrences elevated livet enzymes and elevations to three times the upper limit of normal. however, did not significantly differ from the rate of liver enzyme abnormalities in placebo-treated patients. All abnormáties were reversible anter discontinuation

Cardiovascular - In clinical pharmacology studies, short episodes of asymptomatic ventricular tachycardia occurred in two individuals administered Ave in three untreated subjects.

howed shocreased libido were reported with equal treevency to patients who received Axid and by those given placebo. Rare reports of gynecomastia occurred. Hematologic - Fatal thrombocytopenia was reported in a patient who was treated with Axid and another $\mathrm{H}_{2}$-receptor antagonist. On previous occasions. this patient had experienced thrombocylopenia while taking other drugs. Integumental-Sweating and urticaria were reported significantly more frequently in nizatidine than in placebo patients. Rash and extoliative dermatitis were also reported.

reported. Overdosage: There is little clinical experience with overdosage of Axid in humans. If overdosage occurs, use of activated charcoal, emesis, or lavage Renal dialysis for four to six hours increased plasma ciearance by approximately Test animals that received large doses of nizatidine have exhibited cholinergictype effects, including lacrimation, salivation, emesis, miosis, and diarthe Singie oral doses of $800 \mathrm{mg} k \mathrm{~kg}$ in dogs and of mo/kg respectively so vV 2091 AMP [041288] Axids (nizatidine, Lilly)

Eli Lilly and Company Leely Indianapolis, Indiana

NZ-2901-T-849340 O 1988, ELILLLY, 
$6+183+83+4$ 